\title{
Designing a Novel Multi-epitope Peptide Vaccine Against Pathogenic Shigella spp. Based Immunoinformatics Approaches
}

\author{
Ibrahim Farhani $^{1} \cdot$ Navid Nezafat $^{2,3} \cdot$ Shirin Mahmoodi $^{1}$
}

Accepted: 19 March 2018 / Published online: 11 May 2018

(c) Springer Science+Business Media, LLC, part of Springer Nature 2018

\begin{abstract}
Shigella spp. causes severe diarrhea and dysenteric disease, which known as shigellosis. Until now, no licensed vaccine is available for shigellosis; therefore, development of a novel strategy to produce an effective vaccine is urgently needed. Shigella invasion plasmid antigens (Ipas) such as, Ipa B and Ipa D are conserved, cross-reactive and highly immunogenic antigens among Shigella spp. Outer membrane protein (Omp) A family of Shigella spp. incites effective immune; therefore, Ipa B, Ipa D and Omp A are superb candidate antigens for designing a cross-protective vaccine against Shigella. In this study, different immunoinformatics approaches were used to design a novel multi-epitope peptide vaccine against Shigella spp. $\mathrm{CD}^{+} \mathrm{T}$ cell immune response has an essential role in eradication of shigellosis; in this regard, Ipa B, Ipa D and Omp A were analyzed to identify Helper T cell lymphocytes (HTL) epitopes by various immunoinformatics servers. D0 and D1 flagellin domains of salmonella enteric were used as Toll like receptor 5 (TLR5) agonist which plays a role as mucosal adjuvant. All mentioned segments were fused together by proper amino acid linkers. Linear and conformational B cell epitopes were also determined in vaccine construct. Totally, we believe that the designed vaccine candidate is able to induce cellular and humoral as well as mucosal secretory $\operatorname{IgA}(\operatorname{sIg} \mathrm{A})$ immunity against shigellosis.
\end{abstract}

Keywords Diarrhea $\cdot$ Shigellosis $\cdot$ Vaccine $\cdot$ Epitope $\cdot$ Immunoinformatics

\section{Introduction}

Shigella spp. are gram-negative, immobile and facultative anaerobic bacteria (Killackey et al. 2016). The pathogen is limited to human and primate (Mallett et al. 1993). Global reports indicate that shigellosis leads to about 169 million morbidities in developing countries (Killackey et al. 2016). According to their Lipopolysaccharide (LPS) chain, it has been divided into four pathogenic species, including:(Livio et al. 2014) Shigella flexeneri; the most affluent species, Shigella sonnei; the major agent for shigellosis in the industrial

Shirin Mahmoodi

Sh.mahmoodi@fums.ac.ir

1 Department of Medical Biotechnology, School of Medicine, Fasa University of Medical Sciences, Fasa, Iran

2 Pharmaceutical Sciences Research Center, Faculty of Pharmacy, Shiraz University of Medical Sciences, Shiraz, Iran

3 Department of Pharmaceutical Biotechnology, School of Pharmacy, Shiraz University of Medical Sciences, Shiraz, Iran countries, Shigella dysenteriae that causes pandemic dysentery, and Shigella boydii, which causes $7 \%$ of the shigellosis in less-developed countries (Kotloff et al. 1999). In the context of infection mechanism, Shigella spp. is bound and enter into $\mathrm{M}$ cell (an epithelial cell in the intestine) by type three secretory system (TTSS) (Phalipon and Sansonetti 2007), then propagates in the host epithelial cells and spreads to adjacent cells by Viral G (Vir G) protein (Mani et al. 2016). Another invasion mechanism which is used by Shigella spp. is Shiga toxin that is comprised of A and B-subunits (Gupta et al. 2011). The toxin is bound to Glycosphingolipid (GM) in the intestinal cell membrane via B-subunit, which allows penetrating of A-subunit into the host cell and subsequently leads to inhibit translation process by the ribosome inactivating (Falguieres et al. 2001). Nowadays, the appearance of antibiotics resistant strains has been caused serious concerns about shigellosis treatment, all over the world (Seidlein et al. 2006). Scientific evidence demonstrates that different vaccines have been used against Shigella bacteria (Mani et al. 2016). Killed and inactivated bacteria are the important types of vaccine that are used against Shigella infection, and they are able to stimulate immune system effectively; 
(Ashkenazi and Cohen 2013; McKenzie et al. 2006) but they have some disadvantages such as reactogenicity, instability in different storage condition and limited protection period (Nezafat et al. 2016). Until now, no approved prophylactic or therapeutic vaccine against shigellosis is available on the market (Ashkenazi and Cohen 2013). In this regard, there is an urgent need to find a new approach to struggle with these pathogenic bacteria. Multi-epitope peptide vaccines consisting of cytotoxic T lymphocytes (CTL) and helper T lymphocytes (HTL) epitopes that are designed by immunoinformatics tools are considered as an important medication against pathogenetic bacteria like Staphylococcus aureus, Helicobacer pylori, Kelebsiella pneumonia, Shigella spp, Human papillomavirus and Vibrio cholerae (Hajighahramani et al. 2017; Nezafat et al. 2016, 2017; Sette et al. 2001; Mahmoodi et al. 2017; Shahbazi et al. 2016; Farhadi et al. 2015). Some advantages such as high specificity, cost-effective production and stability in various conditions outstand these vaccines compare to others (Sette et al. 2001). Low immunogenicity and enzymatic degradation in serum are some disadvantages of epitope vaccines (Mahmoodi et al. 2016). For designing an effective multi-epitope peptide vaccine, dominant epitopes are selected from immunogenic proteins. Invasion plasmid antigens (Ipas) are important effectors in TTSS and considered as protective antigens (Martinez-Becerra et al. 2013). Ipa proteins are expressed by all virulent Shigella serotypes (Levine et al. 2007). Ipa D controls the formation of a tip needle for TTSS (Martinez-Becerra et al. 2013) and Ipa B interact with the host cell membrane to form a pore in it (Blocker et al. 1999). In this regard, Ipa proteins can be considered as ideal vaccine candidate antigens. Outer membrane proteins (Omps) which are immunogen and cross-reactive among Shigella serotypes, are another vaccine target (Pore et al. 2011; Pore and Chakrabarti 2013). Omp A is considered as protective, cross-reactive and conserved antigen among Shigella spp. and because of its location on the cell surface, it is highly immunogen (Pore et al. 2011). Adjuvants are one of the important components in a vaccine construct that stimulate the immune system and modulate it towards the desired immune responses (HTL or CTL) (Apostólico 2016). Due to the low immunogenicity of multi-epitope peptide vaccines, adjuvants are incorporated into vaccine construct (Smith et al. 2003). Toll-like receptors (TLRs) are conserved receptors, expressed on the immune and nonimmune cells (Kumar et al. 2009) and known as a class of the pathogen recognition receptors (PRR) which recognize specific molecules of pathogens as an agonist (Smith et al. 2003). Bacterial flagellin, as a TLR5 agonist, is able to efficiently activate both innate and adaptive immune systems via binding to TLR5; therefore it has been used as an effective mucosal vaccine adjuvant in different researches (Honko et al. 2006; Mizel et al. 2009; Mori et al. 2012). In this study, we harnessed different bioinformatics approaches for designing a novel multi-epitope peptide vaccine against pathogenic Shigella spp. Our vaccine contains several T-cell, $\mathrm{B}$ cell and interferon gamma inducing epitopes. In order to enhance vaccine immunogenicity D0 and D1 flagellin domains were used as a strong natural adjuvant bound to TLR5.

\section{Methods}

The overall procedures used for vaccine design is demonstrated in Fig. 1.

\section{Sequence Retrieval}

Related sequences of Ipa B (Accession no P18011), Ipa D (Accession no. P18013), Omp A (Accession no. Q7X0I7) and flagellin of salmonella enteric subs. Enteric serovar Dublin were retrieved from the universal protein resource (uniprot) database (http://www.uniprot.org/).

\section{Immunoinformatics Analyses}

\section{MHC-II Binding Epitope Prediction}

MHC-II binding epitopes were selected via four different servers (IEDB, MHCpred, MHC2pred and RANKPEP). Mouse MHC-II alleles, namely H-2IAb, H-2IAd, and $\mathrm{H}$-2IAs were chosen for MHC-II binding epitopes prediction. The IEDB at (http://tools.iedb.org/mhci/) employs four prediction methods, including consensus method, sturniolo's method, stabilized matrix method (SMM)-align and average relative binding (ARB) for MHC-II binding epitope prediction (Zhang et al. 2008). MHC-II binding epitope selection was also performed by MHCpred at http://www.ddgpharmfa c.net/mhopred/MHCPred/, which is based on allele specificquantitative structure activity relationship (QSAR) models (accuracy about 90\%) (Guan et al. 2003). The MHC2pred uses support vector machine (SVM) method with the accuracy of about $80 \%$ for 42 alleles in MHC-II binding epitope selection (Bordner and Mittelmann 2010). RANKPEP at http://imed.med.ucm.es/Tools/rankpep.html predicts MHCII binding epitopes based on the position specific scoring matrices (PSSMs); the binding threshold for the top scoring MHC-II epitopes is 4-6\% (Reche et al. 2002).

\section{Linear B-Cell Epitope Prediction}

Three servers (ABCpred, BCpred and BepiPred) were used for linear B-cell epitope prediction. The ABCpred at http:// www.imtech.res.in/raghava/abcpred/ predicts linear B cell epitopes by artificial neural network (ANN) which is based on applying fixed length patterns; the accuracy of ABCpred 
Fig. 1 A summary of the methods that were employed for in silico design of multi-epitope peptide vaccine

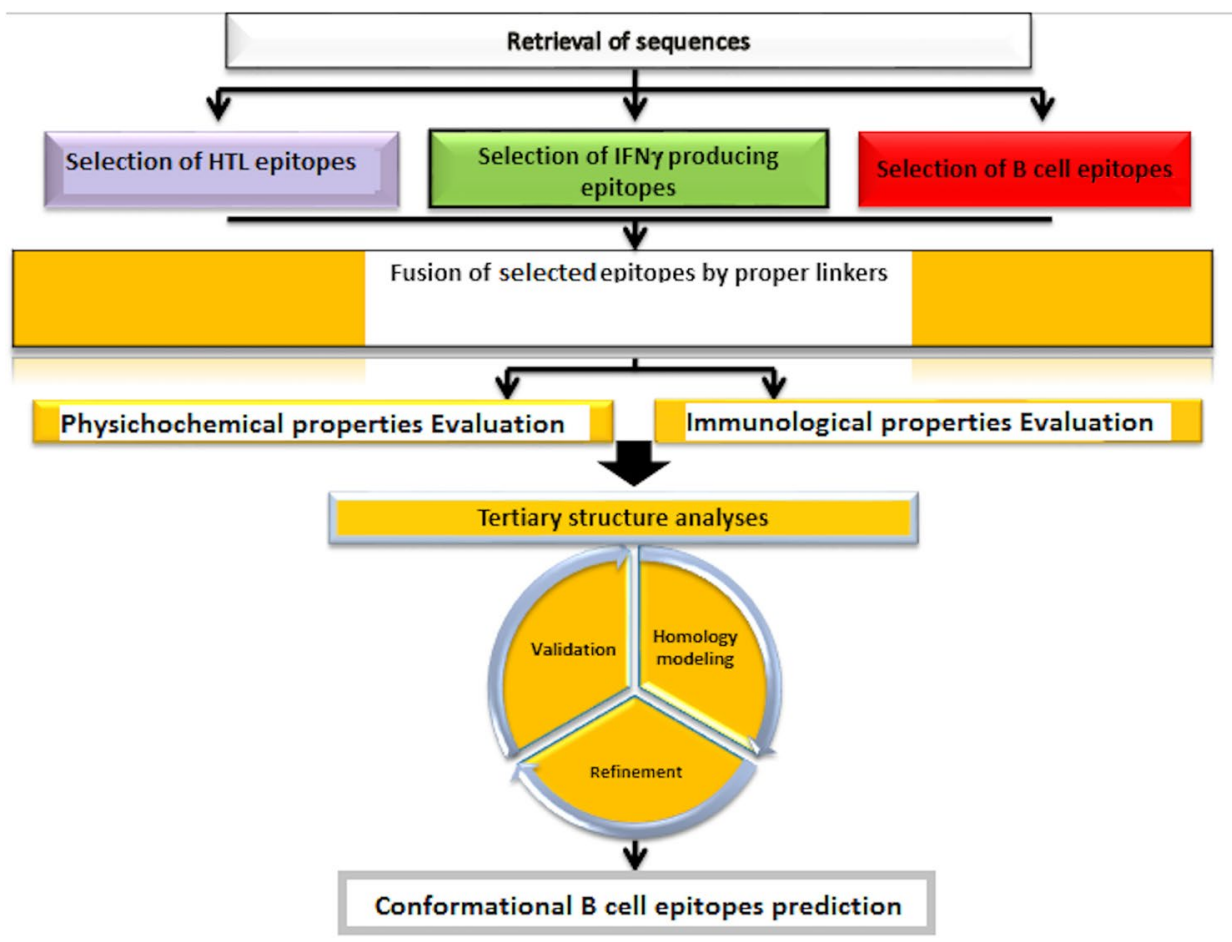

is $65.93 \%$, and the default threshold 0.51 was used (Thomas et al. 2004). The BCpred server at http://ailab.ist.psu.edu/ bcpred/predict.html uses subsequences kernel (SSK) and a support vector machine (SVM) with accuracy and specificity, 74.57 and 75\% respectively (EL-Manzalawy et al. 2008). BepiPred at http://www.cbs.dtu.dk/services/BepiPred/ combines hidden markov model and a propensity scale method for prediction of linear B cell epitopes. The score threshold is $0.35 \%$ (Larsen et al. 2006).

\section{Selection of the Epitope Segments}

The obtained results were compared together, and the regions with the highest overlaps were determined. Selected epitopes can induce various types of immune responses, including HTL, B cell and INF-gama responses.

\section{Prediction of Interferon-Gamma Inducing Epitopes}

For designing an effective vaccine, interferon-gamma inducing epitopes from the selected MHC-II binding epitopes were predicted. IFNepitope server at http://crdd.osdd.net/ raghava/ifnepitope/ employs a dataset that composed of 3705 IFNepitope inducing and 6728 non-inducing MHC class II binders (Dhanda et al. 2013). This web server predicts interferon-gamma inducing epitopes based on three major modules, including predict, design and scan.

\section{Vaccine Properties Evaluation}

\section{Antigenicity Evaluation}

To evaluate the antigenicity of final vaccine construct two servers were used. ANTIGENpro is available at http://scrat ch.proteomics.ics.uci.edu/ that predicts antigenicity based on the results obtained by protein microarray data analysis. ANTIGENpro is a sequence-based, alignment free and pathogen independent software. The accuracy is 75.51 with a prediction threshold of 0.5 (Magnan et al. 2010). Another server is Vaxijen v2.0 that predicts antigenicity based on the target organism such as bacterial, viral and tumor antigen. The server uses a new alignment-independent approach for antigenicity prediction. It's accuracy varies from 70 to $89 \%$ according to target organism (Doytchinova and Flower 2007).

\section{Allergenicity Evaluation}

To evaluate the allergenicity of the designed vaccine two servers, SDAP and PREAL, were used. Cross-referenced accesses to the sequences, structures and IgE epitopes of allergenic proteins were provided by the structural database of allergenic proteins (SDAP) at https://fermi.utmb.edu/ (Ivanciuc et al. 2003). PREAL (Prediction of Allergenic Proteins) server at http://gmobl.sjtu.edu.cn/PREAL/index.php predicts allergenicity based on different biochemical and physicochemical properties of proteins, sequential features 
and subcellular locations with $93.42 \%$ accuracy (Roden et al. 2000).

\section{Physicochemical Properties Evaluation}

The physicochemical features of designed vaccine, including molecular weight (Mw), theoretical pI, instability index, half-life, total number of positive and negative residues and grand average of hydropathicity (GRAVY) were calculated by protparam tool (http://web.expasy.org/protparam) (Gasteiger 2005).

\section{Tertiary Structure Analysis}

\section{Homology Modeling}

I-TASSER server at http://zhanglab.ccmb.med.umich.edu/ITASSER/ was used for homology modeling. The modeling process is performed in four steps: (1) Threading template identification (To identify structural templates based on the query protein sequence), (2) Monte Carlo simulation method and ab initio modeling, (3) Model selection and refinement [selection of the model by structure decoys and refinement using fragment-guided molecular dynamics simulation (FGMD)], (4) Structure-based function annotation was performed via applying $\mathrm{COACH}$ approach. The obtained models are identified with a confidence score, known as C-score (the higher the value indicates the higher the confidence) (Yang et al. 2015).

\section{Refinement of the 3D Modeled Structure}

Two servers: GalaxyRefine and 3Drefined were used to refine the best 3D model resulted from I-TASSER. GalaxyRefine server at http://galaxy.seoklab.org/cgi-bin/submit.cgi, applies mild and aggressive relaxation methods to refine the 3D model. Mild relaxation method refines side chains while secondary structure segments and loops are refined by the aggressive method (Shin et al. 2014). The 3Drefined at http://sysbio.rnet.missouri.edu/3Drefine/ is a server for efficient protein structure refinement. This server optimizes hydrogen binding network and performs atomic-level energy minimization (Bhattacharya et al. 2016).

\section{Validation of the 3D Structure}

3D refined vaccine structures were validated using Verify3D and ERRAT servers. The ERRAT server (http://servi ces.mbi.ucla.edu/ERRAT/) performs differentiating between correctly and incorrectly regions of protein structures based on non-bonded atomic interactions (Colovos and Yeates 1993). Verify 3D (http://services.mbi.ucla.edu/Verify3D/) was used to validate the $3 \mathrm{D}$ structure of vaccine through measuring the compatibility of the 3D protein model with its sequence (Lüthy et al. 1992).

\section{Prediction of Conformational B-Cell Epitopes}

Conformational B-cell epitopes were predicted by DiscoTope server from the 3D model. DiscoTope method is based on the protein structure and epitope propensity scores. It's sensitivity and specificity are 0.47 and 0.75 respectively, the default threshold (-3.7) (Kringelum et al. 2012).

\section{In Silico Cloning}

To perform cloning and expression of protein vaccine in a vector, the reverse translation and codon optimization of vaccine protein sequence were performed by Codon Usage Wrangler server (http://www.mrc-lmb.cam.ac.uk/ms/metho ds/codon.html), also using the GenScript Rare Codon Analysis Tool (https://www.genscript.com/tools/rare-codon-analy sis), different features of sequences, including Codon Adaptation Index (CAI), GC content, and Codon Frequency Distribution (CFD) were evaluated.

\section{Results}

\section{Immunoinformatics Assays}

\section{MHC Class II Binding Epitopes}

Ipa B, Ipa D and Omp A were used for MHC class II binding epitopes prediction by four different servers (IEDB, MHCpred, MHC2pred and RANKPEP) (Tables 1, 2, 3). Also, the high-scored and overlapped regions between selected epitopes were determined (Table 4).

\section{Interferon-Gamma Inducing Epitopes and Linear B Cell Epitopes Prediction}

The predicted peptide segments were submitted to the IFNepitope server to project interferon-gamma inducing epitopes (Table 5). Linear B cell epitopes were selected among the predicted HTL epitopes by ABCpred, BCpred and BepiPred; the results are demonstrated in Table 6.

\section{Evaluation of Physicochemical and Immunological Properties of the Designed Vaccine}

The physicochemical properties of the designed vaccine such as molecular weight, amino acid composition, instability index, half-life, aliphatic index, theoretical pI and GRAVY, as well as the immunological properties, including antigenicity and allergenicity are shown in Table 7. 
Table 1 Prediction of HTL epitopes from Ipa B by four different servers

\begin{tabular}{|c|c|c|}
\hline Server & Start-end position & $\begin{array}{l}\text { Best ranked } \\
\text { epitope in the } \\
\text { region }\end{array}$ \\
\hline \multirow[t]{2}{*}{ IEDB } & & Percentile rank $\mathrm{k}^{\mathrm{a}}$ \\
\hline & $322-354$ & 2.99 \\
\hline \multirow[t]{4}{*}{ MHC2pred } & & Score $^{b}$ \\
\hline & $395-421$ & 0.85 \\
\hline & 90-104 & 0.71 \\
\hline & $436-448$ & 0.12 \\
\hline \multirow[t]{9}{*}{ MHCpred } & & $-\log \mathrm{IC} 50^{\mathrm{c}}$ \\
\hline & $546-562$ & 8.4 \\
\hline & $3-117$ & 8.15 \\
\hline & $158-183$ & 7.6 \\
\hline & $238-280$ & 7.25 \\
\hline & $571-580$ & 7.07 \\
\hline & $288-541$ & 6.99 \\
\hline & $121-133$ & 6.6 \\
\hline & 190-206 & 6.16 \\
\hline \multirow[t]{8}{*}{ RANKPEP } & & Score $^{b}$ \\
\hline & $482-521$ & 19.23 \\
\hline & $135-145$ & 15.06 \\
\hline & $67-76$ & 10.96 \\
\hline & $292-301$ & 10.78 \\
\hline & $334-357$ & 10.43 \\
\hline & $412-433$ & 9.56 \\
\hline & $533-545$ & 9.06 \\
\hline
\end{tabular}

${ }^{\mathrm{a}}$ Low percentile rank $=$ good binder

${ }^{\mathrm{b}}$ High score $=$ good binder

${ }^{\mathrm{c}}$ High $-\log \mathrm{IC}_{50}=$ good binder

\section{Homology Modeling}

The 3D structure model of the designed multi-epitope peptide vaccine was obtained from the I-TASSER server. Five models with the $\mathrm{C}$-score value in the range of $[-5,2]$ were suggested by the server. The model with the higher $\mathrm{C}$-score is the best model; therefore, a model with $\mathrm{C}$-score value 0.11 was selected for more evaluations.

3D Structure Validation The potential errors and quality of the initial and refined 3D model were evaluated by ERRAT and Verify 3D servers. The ERRAT results demonstrated that the overall quality factor of the initial 3D model is 88.512 (Fig. 2a) and based on Verify 3D score, $57.43 \%$ of residues had an average 3D-1D score greater than 0.2 (Fig. 3a). Among the refined models, which were refined by GalaxyRefine, model no. 3 was selected as the best 3D model; the ERRAT factor and Verify 3D score residues of the final model were calculated 94 and $65 \%$, respectively (Figs. 2b, 3b); therefore, this 3D model was used for the fol-
Table 2 Prediction of HTL epitopes from Ipa D by four different servers

\begin{tabular}{|c|c|c|}
\hline Server & Start-end position & $\begin{array}{l}\text { Best ranked } \\
\text { epitope in the } \\
\text { region }\end{array}$ \\
\hline \multirow[t]{4}{*}{ IEDB } & & Percentile rank ${ }^{\mathrm{a}}$ \\
\hline & $69-76$ & 1.98 \\
\hline & $127-145$ & 3.31 \\
\hline & $293-311$ & 6.71 \\
\hline \multirow[t]{4}{*}{ MHC2pred } & & Score ${ }^{b}$ \\
\hline & $68-81$ & 0.85 \\
\hline & $189-213$ & 0.82 \\
\hline & $222-235$ & 0.53 \\
\hline \multirow[t]{5}{*}{ MHCpred } & & $-\log \mathrm{IC} 50^{\mathrm{c}}$ \\
\hline & $214-331$ & 7.95 \\
\hline & 159-198 & 7.32 \\
\hline & $1-83$ & 6.45 \\
\hline & $87-125$ & 6.25 \\
\hline \multirow[t]{5}{*}{ RANKPEP } & & Score $^{\mathrm{b}}$ \\
\hline & $112-120$ & 19.88 \\
\hline & $160-176$ & 14 \\
\hline & $63-76$ & 12.71 \\
\hline & $242-258$ & 12.61 \\
\hline
\end{tabular}

${ }^{a}$ Low percentile rank $=$ good binder

${ }^{\mathrm{b}}$ High score $=$ good binder

${ }^{\mathrm{c}} \mathrm{High}-\mathrm{Log} \mathrm{IC}_{50}=$ good binder

Table 3 Prediction of HTL epitopes from Omp A by four different servers

\begin{tabular}{lcl}
\hline Servers & Start-end position & $\begin{array}{l}\text { Best ranked } \\
\text { epitope in the } \\
\text { region }\end{array}$ \\
\hline IEDB & $252-271$ & $\begin{array}{l}\text { Percentile rank } \\
\text { a }\end{array}$ \\
MHC2pred & $113-127$ & 2.23 \\
& $316-327$ & Score \\
& $177-207$ & 0.78 \\
MHCpred & $7-327$ & 0.63 \\
RANKPEP & & 0.46 \\
& $70-83$ & $-{\text { Log IC } 50^{\mathrm{c}}}^{\mathrm{b}}$ \\
& $258-271$ & 7.29 \\
& $143-155$ & Score \\
& $168-200$ & 14.15 \\
& $99-111$ & 12.39 \\
& & 10.66 \\
\end{tabular}

${ }^{\mathrm{a}}$ Low percentile rank $=$ good binder

${ }^{\mathrm{b}}$ High score $=$ good binder

${ }^{\mathrm{c}} \mathrm{High}-\mathrm{Log} \mathrm{IC}_{50}=$ good binder 
Table 4 Final selected HTL epitopes from Omp A, Ipa D, and Ipa B antigens

\begin{tabular}{|c|c|c|c|}
\hline Protein & \multicolumn{2}{|c|}{ Start-end position } & HTL epitopes \\
\hline \multirow[t]{3}{*}{ Omp A } & $113-127$ & & GASFKDHDTGVSPVF \\
\hline & $143-155$ & & LEYQWTNNIGDAN \\
\hline & $177-207$ & & GEAAPVVAPAPAPEVQTKHFTLKSDVLFNFN \\
\hline \multirow[t]{6}{*}{ Ipa D } & $68-81$ & & KTLTKTSLEEIALH \\
\hline & $112-120$ & & ELLHSAPKE \\
\hline & $160-176$ & & YTQMYQDFSAVLSSLAG \\
\hline & $222-235$ & & QANKWLTELGGTIG \\
\hline & $242-258$ & & GYVVSINMTPIDNMLK \\
\hline & $293-311$ & & NLQTLVQKYSNANSIFDNL \\
\hline \multirow[t]{7}{*}{ Ipa B } & $67-76$ & & LKAPKSLNAS \\
\hline & $90-104$ & & LGEKSLTALTNKITA \\
\hline & 292-301 & & EYAAEVRKAE \\
\hline & $334-357$ & & LALAAVGLALMVTDAIVQAATGNS \\
\hline & $395-433$ & & $\begin{array}{l}\text { KAKMIGSILGAIAGALVLVAAVVLVATVG- } \\
\text { KQAAAKLAEN }\end{array}$ \\
\hline & $436-448$ & & KIIGKTLTDLIPK \\
\hline & $533-545$ & & EQLSKYISEAIEK \\
\hline Protein & Start-end position & IFN gamma score ${ }^{a}$ & Sequence \\
\hline Omp A & $177-206$ & +1 & GEAAPVVAPAPAPEVQTKHFTLKSDVLFNF \\
\hline \multirow[t]{3}{*}{ Ipa D } & $159-176$ & +2 & SYTQMYQDFSAVLSSLAG \\
\hline & $242-258$ & +0.37 & GGYVVSINMTPIDNMLK \\
\hline & $293-311$ & +0.26 & NLQTLVQKYSNANSIFDNL \\
\hline Ipa B & 292-301 & +0.43 & EYAAEVRKAE \\
\hline
\end{tabular}

${ }^{\text {a Positive }}$ score $=$ the epitope potentiality to inducing IFN gamma secretion
Table 5 Analysis of selected HTL epitopes potentiality are able to inducing IFN gamma secretion, by IFNepitope server lowing studies (Fig. 4). According to the above-mentioned results, the quality of the 3D structure was improved after refinement. The initial and refined 3D models are compared in Fig. 5.

\section{Prediction of Conformational B-cell Epitopes}

Conformational B cell epitopes were determined by Discotope server. Out of 404 total residues, 76 conformational B-cell epitope residues were identified in the final vaccine construct (Table 8).

\section{In Silico Cloning}

Reverse translation and codon optimization of the designed vaccine construct were performed by the Wrangler server. The important properties of the gene sequence in order to achieve a high-level protein expression in the E. coli host, including Codon Adaptation Index (CAI), GC content, and Codon Frequency Distribution (CFD), were determined by the GenScript Tool. CAI of optimized nucleotide sequence was 1 , a CAI of $>0.80$ is considered proper for expression in the selected host (Fig. 6a). The average GC content of
Table 6 Prediction of linear B cell epitopes by three different servers (BCpred, ABCpred, BepiPred)

\begin{tabular}{lcl}
\hline Proteins & Start-end position & $\begin{array}{l}\text { Best ranked epitope } \\
\text { in the region }\end{array}$ \\
\hline Omp A & & Score \\
& $145-203$ & 0.98 \\
& $278-325$ & 0.96 \\
& $14-76$ & 0.91 \\
& $240-259$ & 0.77 \\
Ipa D & $102-126$ & 0.69 \\
& & Score \\
& $200-219$ & 0.98 \\
& $231-259$ & 0.93 \\
& $126-141$ & 0.93 \\
& $174-194$ & 0.84 \\
& $268-310$ & 0.81 \\
& $5-49$ & 0.75 \\
& $57-119$ & 0.57 \\
& & Score \\
& $1-80$ & 0.98 \\
& $512-554$ & 0.86 \\
& $281-304$ & 0.8 \\
& $476-492$ & 0.76 \\
& $99-272$ & 0.72 \\
\hline
\end{tabular}

${ }^{\mathrm{a}}$ High score $=$ good binder 
Table 7 Physicochemical and immunological properties of designed vaccine construct

\begin{tabular}{ll}
\hline Physicochemical properties & Result \\
\hline Instability Index & 32.06 \\
GRAVY & -0.34 \\
Theoretical pI & 5.01 \\
Aliphatic Index & 87.50 \\
Molecular weight & 42562.27 \\
No. of amino acids & 404 \\
Instability Index & 32.06 \\
Total number of negatively charged residues & 40 \\
Total number of positively charged residues & 33 \\
Immunological properties & \\
Antigenicity & \\
$\quad$ ANTIGENpro & 0.95 \\
$\quad$ VaxiJen & 0.64 probable antigen \\
Allerginicity & \\
$\quad$ SDAP & No-allergen \\
PREAL & Non-allergen \\
\hline
\end{tabular}

our vaccine sequence is $58.55 \%$ (Fig. $6 \mathrm{~b}$ ), the percentage GC content between $30-70 \%$ is optimal. Codons with a frequency distribution (CFD) $<30 \%$ are suitable for transcription and translation with high efficacy. The results of $100 \%$ CFD value was obtained for the sequence (Fig. 6c).

\section{Discussion}

Different species of Shigella bacteria lead to dysenteric and diarrheal disease by invading to intestinal epithelium cells (Kweon 2008). The traditional approach for treating Shigella infection is using of different antibiotics such as Nalidixic acid, Ampicillin and Ciprofloxacin (Kosek et al. 2010); however, nowadays increasing of antibiotic resistance Shigella spp. has been known as a major challenge in the worldwide (Ashkenazi and Cohen 2013). Hence an effective therapeutic approach should be considered for Shigella infection. Until now, several types of Shigella vaccines including live attenuated, polysaccharide conjugated, killed bacteria and recombinant vaccines have been presented against Shigella (Martinez-Becerra et al. 2012). In spite of extensive researches to develop an effective Shigella vaccine, up to date, no licensed vaccine is available on the market, therefore; there is an essential need to search for new generation of vaccines against Shigella (Ashkenazi and Cohen 2013). Multi-epitope peptide vaccines that are designed with the help of bioinformatics tools have the high degree of accuracy (Davies and Flower 2007). Moreover, harnessing bioinformatics approaches for designing multi-epitope vaccines are less expensive and time-saving (Mahendran et al. 2016). These vaccines are used as a novel modality to fight different bacterial pathogens such as Helicobacter pylori, Staphylococcus aureus, Vibrio cholerae,
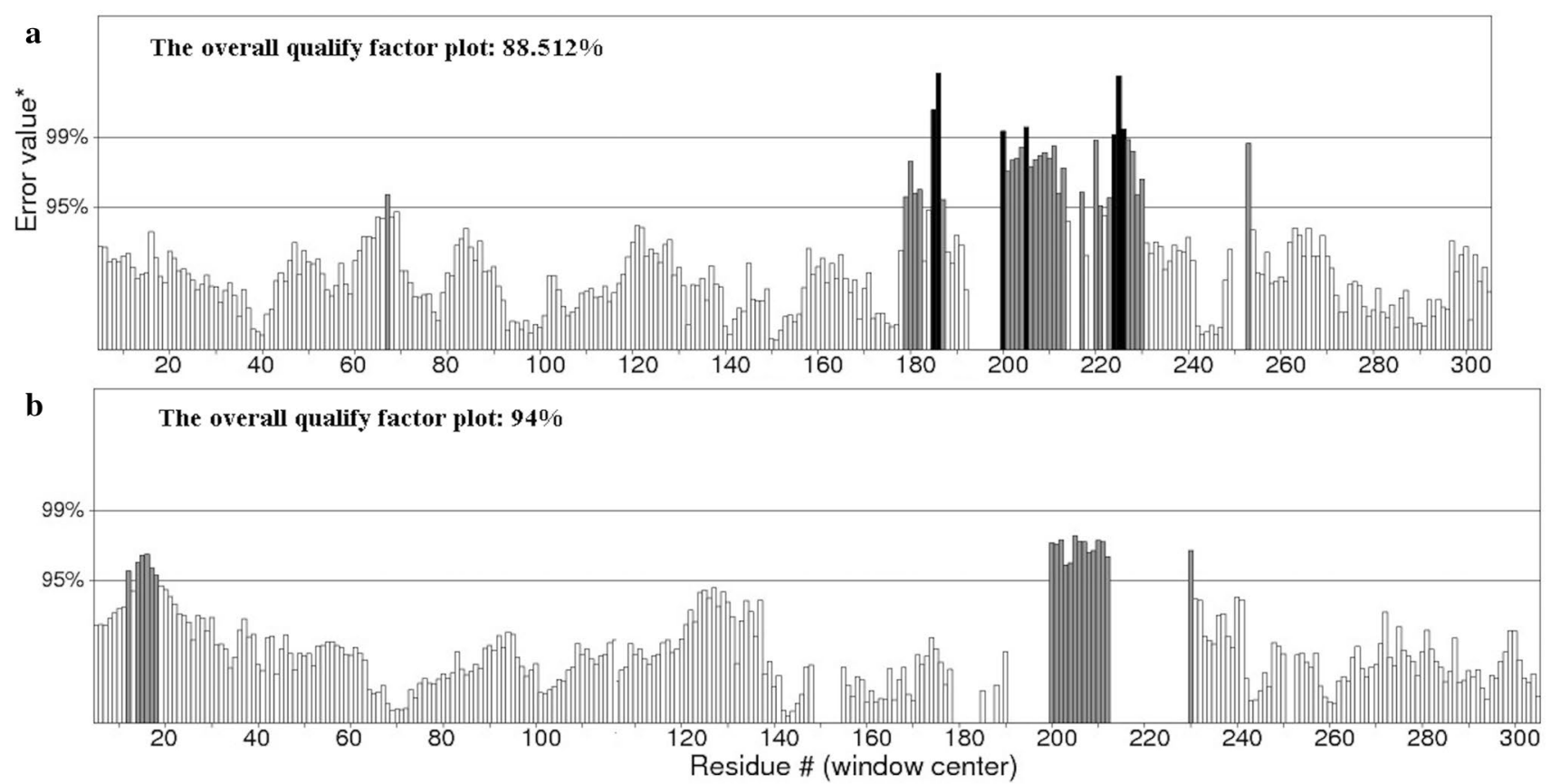

Fig. 2 The overall quality factor plot (ERRAT) of a the initial model is $88.512 \%$ and $\mathbf{b}$ the final model after refinement is $94 \%$; regions of the 3D model that can be rejected at the $99 \%$ level are shown with gray lines in ERRAT plot, and regions that can be rejected at the $95 \%$ confidence are demonstrated with black lines. An overall quality factor value for good high resolution structures is about $95 \%$ or higher 


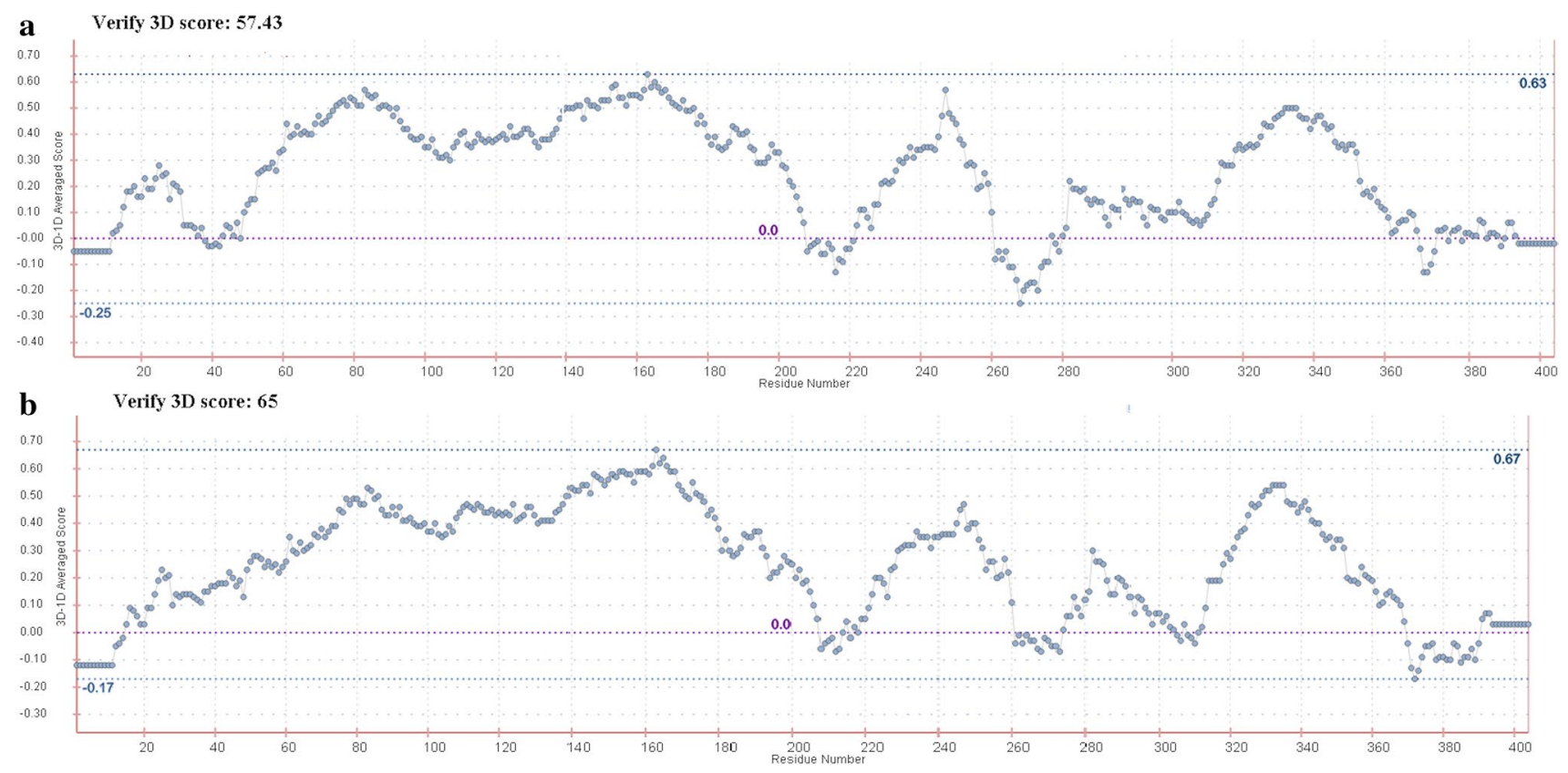

Fig. 3 Verify 3D score of predicted model. The Verify 3D of a the initial model is 57.43 and $\mathbf{b}$ the model after refinement processes is 65 . Reliable residues have an average $3 \mathrm{D}-1 \mathrm{D}$ score greater than zero

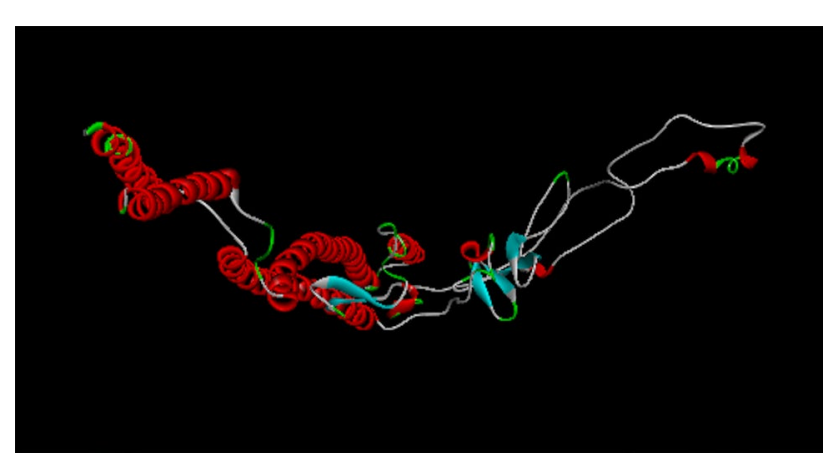

Fig. 4 The refined 3D model of the multi- epitope peptide vaccine. The 3D structure of the designed vaccine was determined by homology modeling using I-TASSER server, and was refined by GalaxyRefine and 3Drefine servers

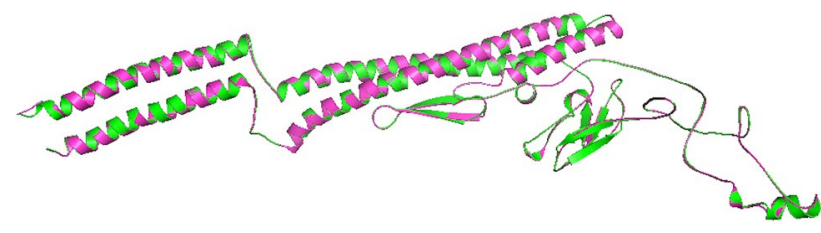

Fig. 5 Superimposition of 3D protein vaccine structure, the initial model and the refined model are colored in magenta and green, respectively. After overall refinement processes, the quality of refined 3D model was improved

Shigella spp. and many others (Nezafat et al. 2016, 2017; Shahbazi et al. 2016; Sharma et al. 2016). As mentioned in introduction, multi-epitope peptide vaccines compare to other types vaccines have some advantages but one of the most important drawbacks related to such vaccine is their low immunogenicity (Groot 2009). To conquer this drawback, adjuvants are added to multi-epitope peptide vaccines construct that play a significant role in stimulation of immune system as well as vaccine delivery (Farhadi et al. 2016; Liu et al. 2008). Toll-like receptors (TLRs) are bound to pathogen-associated molecular patterns (PAMPs) are caused activation of innate immune responses (Duthie et al. 2011). Based on the scientific evidence, bacterial flagellin via binding to TLR5 triggers antigen-presenting cells (APC) to induce innate immune system and acts as a mucosal adjuvant (Lycke 2012). Therefore, vaccines based flagellin adjuvant can be administrated via the nasal and vaginal routes (Moyle 2017). Most experimental evidence suggests that bacterial flagellins consists of four different domains namely D0, D1, D2 and D3, D2 and D3 are considered as the variable and central domains. The D0 and D1 domains of flagellin are located in the $\mathrm{N}$ and $\mathrm{C}$ terminal parts of the flagellin and contribute to TLR5 activation (Smith et al. 2003; Song 2017). Therefore, in this study, D0 and D1 domains of Salmonella enterica subsp. enterica serovar Dublin which have high affinity for binding to TLR5 were used as an adjuvant in vaccine construct. An appropriate vaccine against pathogenic bacteria should provide broad protection against different spp (Martinez-Becerra et al. 2012). Ipa proteins play a critical role in Shigella pathogenesis and are highly conserved among Shigella spp 
Table 8 Identification of conformational B-cell epitopes from 3D protein vaccine structure using DiscoTope

\begin{tabular}{|c|c|c|c|}
\hline Residues number & Amino acid & Contact number & DisCoTope score \\
\hline $38-40$ & $\mathrm{I}-\mathrm{N}-\mathrm{S}$ & $0-11-2$ & $-2.629,-3.275,-2.770$ \\
\hline $43-47$ & $\mathrm{~N}-\mathrm{N}-\mathrm{A}-\mathrm{A}-\mathrm{G}$ & $2-6-4-0-7$ & $-2.350,-2.061,-2.6452 .767,-3.329$ \\
\hline 49 & A & 3 & -3.500 \\
\hline 59 & $\mathrm{~K}$ & 6 & -3.302 \\
\hline $62-63$ & $\mathrm{~T}-\mathrm{Q}$ & $9-10$ & $-2.982,-3.272$ \\
\hline 66 & $\mathrm{R}$ & 9 & -2.722 \\
\hline $101-105$ & $\mathrm{~N}-\mathrm{G}-\mathrm{T}-\mathrm{N}-\mathrm{S}$ & $16-0-2-21-3$ & $-3.349,-1.120,-0.938,-3.291,-0.248$ \\
\hline $107-108$ & $\mathrm{~S}-\mathrm{N}$ & $1-10$ & $-1.772,-2.324$ \\
\hline 111 & $\mathrm{~S}$ & 11 & -2.656 \\
\hline 131 & Q & 5 & -3.137 \\
\hline $133-134$ & $\mathrm{~N}-\mathrm{G}$ & $12-0$ & $-1.952,-1.378$ \\
\hline 143 & Q & 7 & -3.691 \\
\hline 145 & $\mathrm{~K}$ & 7 & -3.341 \\
\hline $151-153$ & $\mathrm{~N}-\mathrm{G}$ & $6-0$ & $-2.882,-0.729,-1.995$ \\
\hline 187-197 & $\mathrm{V}-\mathrm{R}-\mathrm{K}-\mathrm{A}-\mathrm{E}-\mathrm{G}-\mathrm{P}-\mathrm{G}-\mathrm{P}-\mathrm{P}$ & $2-21-7-13-13-15-8-10-0-5-2$ & $\begin{array}{l}-1.138,-3.550,-1.945,-2.530,-2.114 \\
-1.869,-1.096,-0.395,0.634,-1.316 \\
-2.193\end{array}$ \\
\hline 199 & A & 5 & -3.390 \\
\hline $208-214$ & A-P-E-V-Q-T-K & $5-3-9-10-0-0-5$ & $\begin{array}{l}-3.662,-2.362,-3.444,-3.576,-1.741 \\
-1.852,-3.202\end{array}$ \\
\hline 219 & $\mathrm{~K}$ & 0 & -3.570 \\
\hline $224-236$ & F-N-F-G-P-G-P-G-S-Y-T-Q-M-Q & $6-8-0-7-6-16-4-3-2-22-13-12-10$ & $\begin{array}{c}-2.827,-1.634,-0.189,-1.628,-1.850 \\
-2.002,0.618,0.324,0.336,0.618,0.324 \\
0.336,-2.799,-1.248,-1.701,-1.170\end{array}$ \\
\hline 238 & $\mathrm{P}$ & 11 & -3.563 \\
\hline $248-251$ & $\mathrm{~A}-\mathrm{G}-\mathrm{G}-\mathrm{P}$ & $0-6-15-10$ & $-2.269,-2.328,-3.007$ \\
\hline 253 & $\mathrm{P}$ & 9 & -3.015 \\
\hline $263-265$ & $\mathrm{Q}-\mathrm{N}-\mathrm{P}$ & $4-1-0$ & $-2.858,-2.780,-2.920$ \\
\hline 287 & $\mathrm{~T}$ & 2 & -3.343 \\
\hline 301 & $\mathrm{~N}$ & 9 & -3.528 \\
\hline 351 & $\mathrm{~T}$ & 6 & -3.471 \\
\hline 404 & $\mathrm{R}$ & 3 & -3.309 \\
\hline
\end{tabular}

(Turbyfill et al. 2008). Ipa B and D have been demonstrated are able to stimulate immune responses in animal models of Shigella infection (Heine et al. 2013). In addition, antibody against Ipa proteins after human Shigella infection was also detected (Barry et al. 2013), therefore, Ipa proteins can be considered as superb candidate antigens in vaccine development. Another Shigella protective antigen is Omps; Omp A in Shigella flexneri 2a causes to elicit Th1 immune response against shigellosis in animal models (Mukhopadhaya et al. 2006). In this regard, we used Ipa B, Ipa D and Omp A as candidate antigens for epitope selection. Although, antigenspecific $\mathrm{T} \mathrm{CD}^{+}$possesses a primary role in stimulation of adaptive immune system against cytosolic bacterial infection, $\mathrm{T} \mathrm{CD}^{+}$response does not involve in adaptive immune response against Shigella infection (Jehl et al. 2011). Accumulating evidence indicate that, $\mathrm{T} \mathrm{CD}^{+}{ }^{+}$response has a critical role in eradicating of Shigella infection (Mukhopadhaya et al. 2006); therefore immunodominant HTL epitopes were selected from the mentioned antigens (Tables 1, 2, 3, 4). In addition to cellular immune responses, induction of humoral immunity against Shigella infection has an important role in eradication of it, so to reach this goal, linear and conformational B cell epitopes were determined from the antigenic protein sequence and 3D structure of the peptide vaccine respectively (Tables 6,8 ). HTL epitopes activate T-helper cells, including Th1, Th2 and Th17 (Rincón and Flavell 1997; Agnello et al. 2003). Specific type of cytokine is secreted by different type of helper cells, as an example, IFN-gamma that is essential for eradication of intracellular pathogens is released by Th1 (Dhanda et al. 2013); therefore, the presence of IFN-gamma inducing epitopes in selected HTL epitopes were evaluated (Table 5). Linkers are the essential components of the multi-epitope peptide vaccines (Le-Barillec et al. 2005) that have functional 
Fig. 6 Investigation of important parameters of the codonoptimized gene for high-level protein expression in E. coli host. a CAI of gene sequence is 1.00 . A CAI of $>0.8$ is considered as good for expression in selected host. b The average GC content of the sequence is $58.55 \%$. c Codon frequency distribution (CFD) value of gene sequence is $100 \%$ $\mathbf{a}$

Escherichia

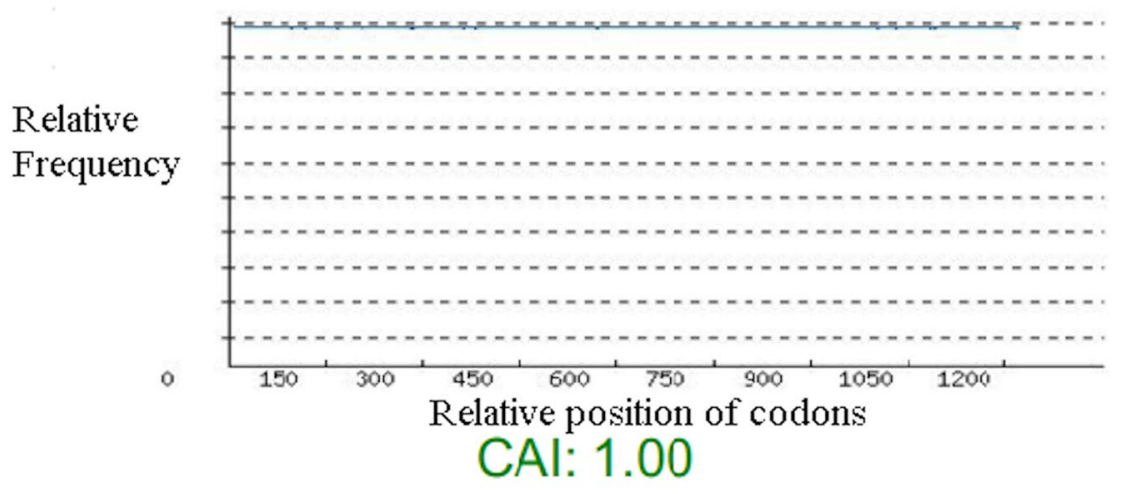

b

\section{GC curve}

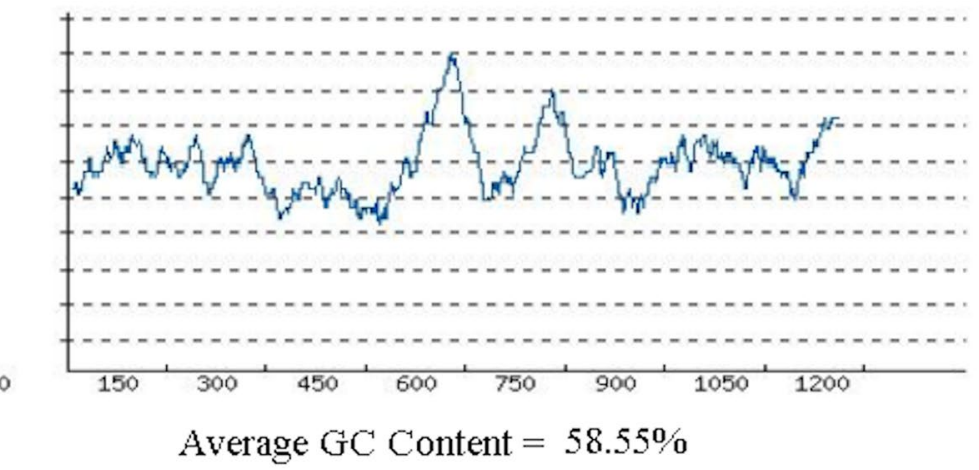

and structural roles in vaccine construct (Bai et al. 2005; Aurora et al. 1997); as an example, EAAAK motif that has $\alpha$-helical structure and is known as a rigid linker, makes distance between domains (Takamatsu et al. 1990). Accordingly, EAAAK was used to join D0 and D1 domains of flagellin to selected HTL epitopes in our designed vaccine construct (Fig. 6). Also, GPGPG motif that is known as a flexible linker and leading enhancement of antigen presentation to MHC II molecules (Livingston et al. 2002) was employed in designed vaccine to link HTL epitopes together (Fig. 6). The important properties of the designed vaccine were evaluated by bioinformatics tool (Table 7). The 3D structure of designed vaccine was determined and evaluated by different servers. Based on the obtained results, the initial 3D model was refined, and then the best refined model was selected (Fig. 4), for identification of conformational B cell epitopes (Table 8). In this study the high immunogenic antigens from Shigella spp. including Ipa B, Ipa D and Omp A were analyzed bioinformaticaly to select HTL and B cell epitopes; furthermore, Flagellin domains were applied as a natural adjuvant. The immunological properties of designed vaccine showed that the designed vaccine is potent immunogen and non-allergen. We expect that our designed multi-epitope peptide candidate vaccine could be able to eradicate shigellosis via triggering cellular and especially humoral immune response. 
Fig. 7 Schematic diagram of vaccine construct consists of adjuvant (D0 and D1 domains of flagellin in $\mathrm{N}$ and $\mathrm{C}$ terminal), HTL epitopes, which were selected from Ipa B, Omp A and Ipa $D$ antigens and fused together by proper linkers

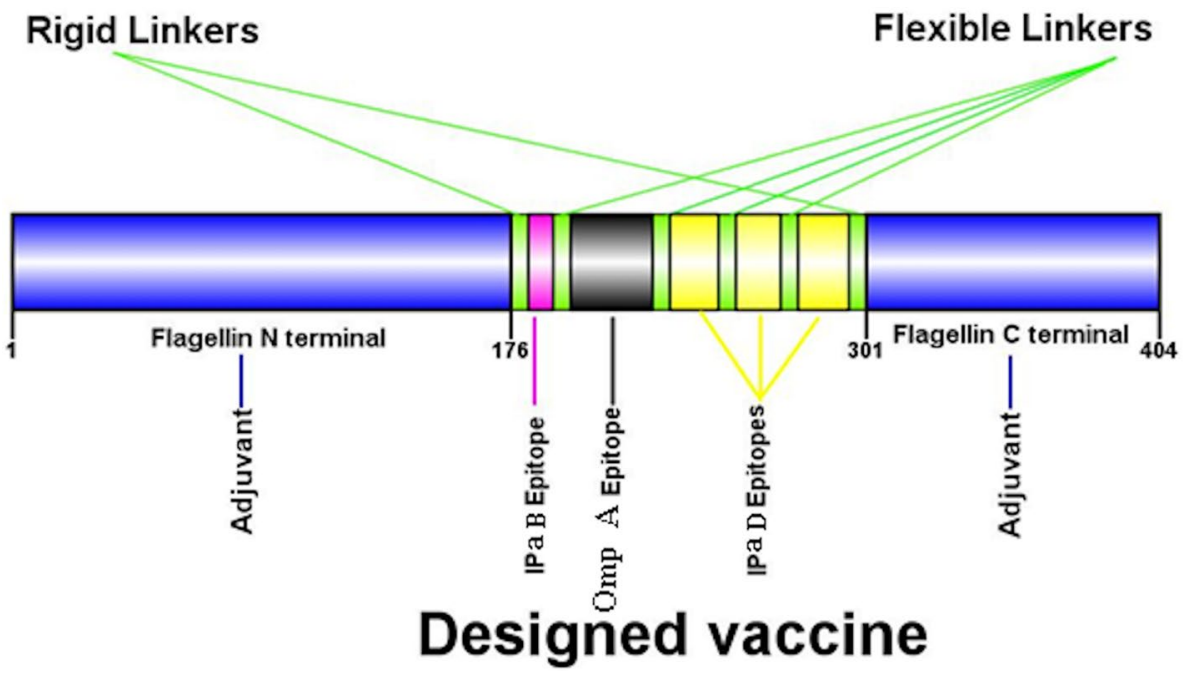

\section{Conclusion}

Developing multi-epitope peptide vaccines against pathogenic bacteria have attracted more attentions in recent years. In this study, we used in silico techniques to design a novel multi-epitope peptide vaccine against Shigells spp. In order to trigger T CD4 ${ }^{+}$immune response, that is critical in fighting Shigella, HTL epitopes were selected from conserved antigens (Ipa B, D) and Omp A in Shigella spp. But in vitro and in vivo immunological tests are needed to evaluate the efficiency of designed multi-epitope peptide vaccine (Fig. 7).

Acknowledgements The authors acknowledge financial support received from the Fasa University of Medical Sciences, Fasa, Iran.

\section{Compliance with Ethical Standards}

Conflict of interest The author(s) confirms that this article content has no conflict of interest.

\section{References}

Agnello D et al (2003) Cytokines and transcription factors that regulate T helper cell differentiation: new players and new insights. J Clin Immunol 23(3):147-161

Apostólico JDS et al (2016) Adjuvants: classification, modus operandi, and licensing. J Immunol Res 2016:1459394

Ashkenazi S, Cohen D (2013) An update on vaccines against Shigella. Ther Adv Vaccines 1(3):113-123

Aurora R et al (1997) Local interactions in protein folding: lessons from the $\alpha$-helix. J Biol Chem 272(3):1413-1416

Bai Y, Ann DK, Shen W-C (2005) Recombinant granulocyte colonystimulating factor-transferrin fusion protein as an oral myelopoietic agent. Proc Natl Acad Sci USA 102(20):7292-7296

Barry EM et al (2013) Progress and pitfalls in Shigella vaccine research. Nat Rev Gastroenterol Hepatol 10(4):245-255

Bhattacharya D et al (2016) 3Drefine: an interactive web server for efficient protein structure refinement. Nucl Acids Res 44(W1):W406-W409
Blocker A et al (1999) The tripartite type III secreton of Shigella flexneri inserts IpaB and IpaC into host membranes. J Cell Biol 147(3):683-693

Bordner AJ, Mittelmann HD (2010) Prediction of the binding affinities of peptides to class II MHC using a regularized thermodynamic model. BMC Bioinform 11(1):41

Colovos C, Yeates TO (1993) Verification of protein structures: patterns of nonbonded atomic interactions. Protein Sci 2(9):1511-1519

Davies MN, Flower DR (2007) Harnessing bioinformatics to discover new vaccines. Drug Discov Today 12(9):389-395

De Groot AS et al (2009) Epitope-based immunome-derived vaccines: a strategy for improved design and safety. In: De Groot AS (ed) Clinical applications of immunomics. Springer, New York, pp 39-69

Dhanda SK, Vir P, Raghava GP (2013) Designing of interferongamma inducing MHC class-II binders. Biol Direct 8(1):30

Doytchinova IA, Flower DR (2007) VaxiJen: a server for prediction of protective antigens, tumour antigens and subunit vaccines. BMC Bioinform 8(1):4

Duthie MS et al (2011) Use of defined TLR ligands as adjuvants within human vaccines. Immunol Rev 239(1):178-196

EL-Manzalawy Y, Dobbs D, Honavar V (2008) Predicting linear B-cell epitopes using string kernels. J Mol Recogn 21(4):243-255

Falguieres T et al (2001) Targeting of Shiga toxin B-subunit to retrograde transport route in association with detergent-resistant membranes. Mol Biol Cell 12(8):2453-2468

Farhadi T et al (2015) Designing of complex multi-epitope peptide vaccine based on omps of Klebsiella pneumoniae: an in silico approach. Int J Pept Res Ther 21(3):325-341

Farhadi T, Ovchinnikov RS, Ranjbar MM (2016) In silico designing of some agonists of toll-like receptor 5 as a novel vaccine adjuvant candidates. Netw Model Anal Health Inform Bioinform 5(1):31

Gasteiger E et al (2005) Protein identification and analysis tools on the ExPASy server. Springer, New York

Guan P et al (2003) MHCPred: a server for quantitative prediction of peptide-MHC binding. Nucleic Acids Res 31(13):3621-3624

Gupta P et al (2011) Recombinant Shiga toxin B subunit elicits protection against Shiga toxin via mixed Th type immune response in mice. Vaccine 29(45):8094-8100

Hajighahramani $\mathrm{N}$ et al (2017) Immunoinformatics analysis and in silico designing of a novel multi-epitope peptide vaccine against Staphylococcus aureus. Infect Genet Evolut 48:83-94

Heine SJ et al (2013) Evaluation of immunogenicity and protective efficacy of orally delivered Shigella type III secretion system proteins IpaB and IpaD. Vaccine 31(28):2919-2929 
Honko AN et al (2006) Flagellin is an effective adjuvant for immunization against lethal respiratory challenge with Yersinia pestis. Infect Immun 74(2):1113-1120

Ivanciuc O, Schein CH, Braun W (2003) SDAP: database and computational tools for allergenic proteins. Nucleic Acids Res 31(1):359-362

Jehl SP et al (2011) Antigen-specific CD8+ T cells fail to respond to Shigella flexneri. Infect Immun 79(5):2021-2030

Killackey SA, Sorbara MT, Girardin SE (2016) Cellular aspects of Shigella pathogenesis: focus on the manipulation of host cell processes. Front Cell Infect Microbiol 6:38

Kosek M, Yori PP, Olortegui MP (2010) Shigellosis update: advancing antibiotic resistance, investment empowered vaccine development, and green bananas. Curr Opin Infect Dis 23(5):475-480

Kotloff KL et al (1999) Global burden of Shigella infections: implications for vaccine development and implementation of control strategies. Bull World Health Organ 77(8):651-666

Kringelum JV et al (2012) Reliable B cell epitope predictions: impacts of method development and improved benchmarking. PLoS Comput Biol 8(12):e1002829

Kumar H, Kawai T, Akira S (2009) Toll-like receptors and innate immunity. Biochem Biophys Res Commun 388(4):621-625

Kweon M-N (2008) Shigellosis: the current status of vaccine development. Curr Opin Infect Dis 21(3):313-318

Larsen JE, Lund O, Nielsen M (2006) Improved method for predicting linear B-cell epitopes. Immunome Res 2(1):2

Le-Barillec $\mathrm{K}$ et al (2005) Roles for $\mathrm{T}$ and NK cells in the innate immune response to Shigella flexneri. J Immunol 175(3): $1735-1740$

Levine MM et al (2007) Clinical trials of Shigella vaccines: two steps forward and one step back on a long, hard road. Nat Rev Microbiol 5(7):540-553

Liu X, Wetzler LM, Massari P (2008) The PorB porin from commensal Neisseria lactamica induces Th1 and Th2 immune responses to ovalbumin in mice and is a potential immune adjuvant. Vaccine 26(6):786-796

Livingston B et al (2002) A rational strategy to design multiepitope immunogens based on multiple Th lymphocyte epitopes. J Immunol 168(11):5499-5506

Livio S et al (2014) Shigella isolates from the global enteric multicenter study inform vaccine development. Clin Infect Dis 59(7):933-941

Lüthy R, Bowie JU, Eisenberg D (1992) Assessment of protein models with three-dimensional profiles. Nature 356(6364):83

Lycke N (2012) Recent progress in mucosal vaccine development: potential and limitations. Nat Rev Immunol 12(8):592-605

Magnan CN et al (2010) High-throughput prediction of protein antigenicity using protein microarray data. Bioinformatics 26(23):2936-2943

Mahendran R et al (2016) Computer-aided vaccine designing approach against fish pathogens Edwardsiella tarda and Flavobacterium columnare using bioinformatics softwares. Drug Des Dev Ther 10:1703

Mahmoodi S et al (2016) Harnessing bioinformatics for designing a novel multi-epitope peptide vaccine against breast cancer. Curr Pharm Biotechnol 17(12):1099-1113

Mahmoodi S et al (2017) Expression and purification of a novel multiepitope peptide vaccine for breast cancer immunotherapy. Minerva Biotecnologica 29(1):1-7

Mallett CP et al (1993) Evaluation of Shigella vaccine safety and efficacy in an intranasally challenged mouse model. Vaccine 11(2):190-196

Mani S, Wierzba T, Walker RI (2016) Status of vaccine research and development for Shigella. Vaccine 34(26):2887-2894
Martinez-Becerra FJ et al (2012) Broadly protective Shigella vaccine based on type III secretion apparatus proteins. Infection immunity 80(3):1222-1231

Martinez-Becerra FJ et al (2013) Parenteral immunization with IpaB/ IpaD protects mice against lethal pulmonary infection by Shigella. Vaccine 31(24):2667-2672

McKenzie R et al (2006) Safety and immunogenicity of an oral, inactivated, whole-cell vaccine for Shigella sonnei: preclinical studies and a Phase I trial. Vaccine 24(18):3735-3745

Mizel SB et al (2009) Flagellin-F1-V fusion protein is an effective plague vaccine in mice and two species of nonhuman primates. Clin Vaccine Immunol 16(1):21-28

Mori J et al (2012) Chimeric flagellin as the self-adjuvanting antigen for the activation of immune response against Helicobacter pylori. Vaccine 30(40):5856-5863

Moyle PM (2017) Biotechnology approaches to produce potent, self-adjuvanting antigen-adjuvant fusion protein subunit vaccines. Biotechnol Adv. https://doi.org/10.1016/j.biotechadv .2017 .03 .005

Mukhopadhaya A, Mahalanabis D, Chakrabarti M (2006) Role of Shigella flexneri 2a 34 kDa outer membrane protein in induction of protective immune response. Vaccine 24(33):6028-6036

Nezafat $\mathrm{N}$ et al (2016) Designing an efficient multi-epitope peptide vaccine against Vibrio cholerae via combined immunoinformatics and protein interaction based approaches. Comput Biol Chem 62:82-95

Nezafat $\mathrm{N}$ et al (2017) Designing an efficient multi-epitope oral vaccine against Helicobacter pylori using immunoinformatics and structural vaccinology approaches. Mol BioSyst 13(4):699-713

Phalipon A, Sansonetti PJ (2007) Shigella's ways of manipulating the host intestinal innate and adaptive immune system: a tool box for survival? Immunol Cell Biol 85(2):119-129

Pore D, Chakrabarti MK (2013) Outer membrane protein A (OmpA) from Shigella flexneri 2a: a promising subunit vaccine candidate. Vaccine 31(36):3644-3650

Pore D et al (2011) Outer membrane protein A (OmpA) of Shigella flexneri $2 \mathrm{a}$, induces protective immune response in a mouse model. PLoS ONE 6(7):e22663

Reche PA, Glutting J-P, Reinherz EL (2002) Prediction of MHC class I binding peptides using profile motifs. Hum Immunol 63(9):701-709

Rincón M, Flavell RA (1997) Transcriptional control in the Th1/Th2 decision. Curr Biol 7(11):R729-R732

Roden RB et al (2000) Minor capsid protein of human genital papillomaviruses contains subdominant, cross-neutralizing epitopes. Virology 270(2):254-257

Sette A et al (2001) The development of multi-epitope vaccines: epitope identification, vaccine design and clinical evaluation. Biologicals 29(3-4):271-276

Shahbazi M et al (2016) In silico sub-unit hexavalent peptide vaccine against an staphylococcus aureus biofilm-related infection. Int J Pept Res Ther 22(1):101-117

Sharma D et al (2016) Immunoinformatic identification of potential epitopes against shigellosis. Int J Pept Res Ther 22(4):481-495

Shin W-H et al (2014) Prediction of protein structure and interaction by GALAXY protein modeling programs. Bio Des 2(1):1-11

Smith KD et al (2003) Toll-like receptor 5 recognizes a conserved site on flagellin required for protofilament formation and bacterial motility. Nat Immunol 4(12):1247-1253

Song WS et al (2017) A conserved TLR5 binding and activation hot spot on flagellin. Sci Rep 7:40878

Takamatsu N et al (1990) Production of enkephalin in tobacco protoplasts using tobacco mosaic virus RNA vector. FEBS Lett 269(1):73-76 
Thomas A, Milon A, Brasseur R (2004) Proteins-structure function and bioinformatics. Wiley, New York

Turbyfill KR, Kaminski RW, Oaks EV (2008) Immunogenicity and efficacy of highly purified invasin complex vaccine from Shigella flexneri 2a. Vaccine 26(10):1353-1364

von Seidlein L et al (2006) A multicentre study of Shigella diarrhoea in six Asian countries: disease burden, clinical manifestations, and microbiology. PLoS Med 3(9):e353
Yang J et al (2015) The I-TASSER Suite: protein structure and function prediction. Nat Methods 12(1):7-8

Zhang Q et al (2008) Immune epitope database analysis resource (IEDB-AR). Nucleic Acids Res 36(suppl_2):W513-W518 